\title{
CHANGES IN FERROELECTRIC PROPERTIES OF MASD ALUM ALONG WITH TEMPERATURE BY USING PLCM MODEL
}

\author{
Anubhuti Mamgain* and Trilok Chandra Upadhyay \\ Department of Physics, H.N.B. Garhwal University, (A Central University), Srinagar (Garhwal) \\ 246174 Uttarakhand
}

Corresponding Author E-mail id: anubhuti.mamgain01@gmail.com

Received: 11.09.2019; Revised: 16.10.2019; Accepted: 10.11.2019

CSociety for Himalayan Action Research and Development

\begin{abstract}
With addition of third-and fourth-order phonon anharmonic interactions, and extra spin-lattice interaction term into pseudospin-lattice coupled mode (PLCM) model, MASD alum has been studied. Employing double time temperature dependent Green's function technique expressions for shift and width of response function, normal mode frequency, dielectric constant and loss stangent have been derived for MASD alum. Numerical calculation has been done. Theoretical results have been compared with experimental results of Pepinsky et al. [16]. A good agreement has been found.
\end{abstract}

Keywords: Ferroelectrics, green's function, dielectric constant, loss tangent, anharmonicity.

\section{Introduction}

Ferroelectric substances have been found useful in electronics and technology. Memory devices, infrared detectors, optical modulators etc. are some examples. Ronald et al. [1] worked on organic non-volatile memory devices based on ferroelectricity and suggested the use of low-cost solution-based processing techniques to make integrated memory circuits that consist of crossbar arrays of ferroelectrics capacitors e.g., by ink-jet printing metal contacts, conductive polymer interface layers etc. Some alums show ferroelectric behaviour.

Methyl ammonium aluminium alum (MASD), $\left(\mathrm{CH}_{3} \mathrm{NH}_{3}\right) \mathrm{Al}\left(\mathrm{SO}_{4}\right)_{2} .12 \mathrm{H}_{2} \mathrm{O}$ becomes ferroelectric below $177 \mathrm{~K}$ temperature. In ferroelectric state $\mathrm{CH}_{3} \mathrm{NH}_{3}$ groups are arranged in dumbbell shape. They are in ordered state. In paraelectric phase they are oriented in disordered fashion. Venkatesh et al. [2] have done spectroscopic studies of ferroelectric MASD alum. They observed that sulphate or selenate ions do not undergo any distortion in low temperature phase. Griffith and Powell [3] have measured dielectric constant at different temperatures. Rao et al. [4] have measured dielectric constant of MASD alum. They observed phase transition near 177K. Derby [5] has done growth studies of different alums. Weber [6] has made dielectric measurements on alums. Boujelbene and Mihiri [7] have done Raman spectroscopic studies on MASD alums. Sachdeva et al. [8] have done growth studies on alums. Gu and $\mathrm{Li}$ [9] have done spectral studies of alums. Gu and Hao [10] have studied electrolytic coloration below $0^{\circ} \mathrm{C}$ and spectral properties of ammonium alum crystals using a pointed anode. Zhang et al. [11] have carried out experimental study on hysteresis curves on alums. Basara et al. [12] have done electric conductivity studies on alums. Singh et al. [13] have done synthesis and characterization studies on alums. Sajan et al. [14] have studied physical properties of polymer mixed alums. Ghanew et al. [15] have studied experimentally effects of doping of different polymers on alums. Pepinsky et al. [16] have measured dielectric constant of MASD alums at different temperatures. They observed that 
dielectric constant reaches maximum at $177 \mathrm{~K}$ anomalously (abruptly) showing phase transition at this temperature. Shaxin et al. [17] have studied application of alum in inorganic synthesis. Petrusevski [18] has carried out experimental study of vibrational spectra of selenate and related alums. Bow et al. [19] have done alums adjuvant action studies. Mansingh et al. [20] have done work on low frequency electrical behaviour of ferroelectric MASD alum. Cha and Strauss [21] together worked on local structure and tunnelling kinetics of ammonium aluminium alum by infrared hole-burning. Hao and Tsu [22] have worked on dimorphism in methylammonium aluminium alum studied by X-ray diffraction and electron paramagnetic resonance. $\mathrm{O}^{\prime}$ Reilly and Tsang [23] have worked on magnetic resonance studies of ferroelectric Methylammonium alum. Chaudhury et al. [24] gave phenomenological explanation of the anomalous dielectric behaviour of alums with pseudo-spin-lattice coupled-mode model.
Theoretical studies to understand phase transition and dielectric behaviour of MASD alums were initiated by Choudhuri et al. [24] They have considered pseudospin-lattice coupled mode model along with fourth-order phonon anharmonic interaction term. They have not considered third-order phonon anharmonic interaction term. They have decoupled correlations at an early stage. In the present work, we have considered pseudo-spin lattice coupled mode model along with third-and fourth-order phonon anharmonic interactions terms. With the help of modified model and double-time Green's function method, expressions for shift, width, ferroelectric mode frequency, dielectric constant and loss tangent have been derived. By fitting model values in expressions temperature dependences of ferroelectric mode frequency, dielectric constant and loss tangent have been calculated, for MASD alum. Theoretical results have been compared with experimental results of Pepinsky et al. [16].

\section{Model Hamiltonian}

For ferroelectric MASD crystal, the pseudo-spin lattice coupled mode model (earlier used by Chaudhury et al. [23] by adding third-and fourth order phonon anharmonic interactions and other terms. The pseudospin lattice coupled mode model is expressed as:

$$
H_{1}=-2 \Omega \sum_{i} S_{i}^{x}-\frac{1}{2} \sum_{i j} J_{i j} S_{i}^{z} S_{j}^{z}+\frac{1}{4} \sum_{i k} \omega_{k}\left(A_{k}^{+} A_{k}+B_{k}^{+} B_{k}\right)-\sum_{i k} V_{i k} S_{i}^{z} A_{k},
$$

we shall add:

$$
H_{2}=\sum_{i j k} V^{(3)}\left(k_{1}, k_{2}, k_{3}\right) A_{k_{1}} A_{k_{2}} A_{k_{3}}+\sum_{k_{1} k_{2} k_{3} k_{4}} V^{(4)}\left(k_{1} k_{2} k_{3} k_{4}\right) A_{k_{1}} A_{k_{2}} A_{k_{3}} A_{k_{4}}-\sum_{i k} V_{i k} S_{i}^{x} A_{k}
$$

where, $\Omega$ is proton tunnelling frequency between double well potential $\mathrm{O}-\mathrm{H} \ldots \mathrm{O}$ bonds, $S_{i}^{x}$ is $\mathrm{x}-$ component of the spin variables, $J_{i j}$ is exchange interaction constant, $S_{i}^{z}$ is z-component of pseudospin variable $\mathrm{S}, V_{i k}$ is spin- lattice interaction constant, $A_{k}$ and $B_{k}$ are position and momenta co-ordinates. $V^{3}\left(k_{1}, k_{2}, k_{3}\right)$ and $V^{4}\left(k_{1}, k_{2}, k_{3}, k_{4}\right)$ are the third and fourth order phonon anharmonic interactions terms. The third term in Eq.(2) describes an indirect coupling between the tunnelling motion of one proton and other proton. This term describes the modulation of distance between the two equilibrium sites in the OH...O bonds. This modulates $\Omega$ by non polar optic phonons. We shall consider the total Hamiltonian:

$$
H=H_{1}+H_{2} \text {, }
$$

for our study of MASD crystal.

\section{Green's function, Width and Shift}


Following Zubarev [25], we consider the Green's function:

$$
\begin{aligned}
G_{i j}\left(t-t^{\prime}\right) & =<<S_{i}^{z}(t) ; S_{j}^{z}\left(t^{\prime}\right)>> \\
& =-i \theta\left(t-t^{\prime}\right)<\left[S_{i}^{z}(t), S_{j}^{z}\left(t^{\prime}\right)\right]>,
\end{aligned}
$$

where $\theta$ is step function, $\theta=0$ for $\mathrm{t}<\mathrm{t}^{\prime}$ and $\theta=1$ for $\mathrm{t}>\mathrm{t}^{\prime}$. Differentiation of Green's function (4) with respect to time $t$ and $t^{\prime}$ two times using model Hamiltonian (3), Fourier transforming and putting into Dyson's equation form:

$$
G_{i j}(\omega)=G_{i j}^{0}(\omega)+G_{i j}^{0}(\omega) \tilde{P}(\omega) G_{i j}^{0}(\omega),
$$

we obtain,

$$
G_{i j}(\omega)=\frac{\Omega<S_{i}^{x}>\delta_{i j}}{\pi\left[\omega^{2}-\widetilde{\Omega}^{2}-\tilde{P}(\omega)\right]},
$$

where,

$$
\begin{aligned}
& G_{i j}^{0}(\omega)=\frac{\Omega<S_{i}^{x}>}{\pi\left(\omega^{2}-4 \Omega^{2}\right)} \\
& \tilde{\Omega}^{2}=\left(J_{i j}<S_{i}^{z}>\right)^{2}+4 \Omega^{2}-2 \Omega J_{i j}<S^{x}>,
\end{aligned}
$$

and,

$$
\tilde{P}(\omega)=\frac{\pi}{\Omega<S_{i}^{x}>}<<F_{i}(t) ; F_{j}\left(t^{\prime}\right)>>,
$$

where,

$$
\begin{aligned}
F_{i}(t) & =-\Omega \sum_{i} \sum_{i j} J_{i j} S_{i}^{x} S_{j}^{z}-\Omega \sum_{i} \sum_{i j} J_{i j} S_{i}^{z} S_{i}^{x}-2 \Omega \sum_{i} \sum_{i k} V_{i k} A_{k} S_{i}^{x}+4 \Omega \sum_{i} \sum_{i k} V_{i k} A_{k} S_{i}^{z} \\
& -\frac{1}{2} \sum_{i k} \sum_{i j} V_{i k} A_{k} J_{i j} S_{i}^{x} S_{j}^{z}-\frac{1}{2} \sum_{i k} \sum_{i j} V_{i k} A_{k} J_{i j} S_{i}^{z} S_{i}^{x}-\sum_{i k} V_{i k}^{2} A_{k}^{2} S_{i}^{x}+\sum_{i k} V_{i k}^{2} A_{k}^{2} S_{i}^{z} .
\end{aligned}
$$

Solving Eq. (8) we obtain:

$\widetilde{\Omega}^{2}=a^{2}+b^{2}-b c$, where:

$a=J<S_{i}^{z}>$

$b=2 \Omega$

$c=J<S_{i}^{x}>$.

$\tilde{P}(\omega)$ contains higher order Green's functions like $<<\mathrm{AB} ; \mathrm{CD}>>,<<\mathrm{ABC} ; \mathrm{DEF}>>$. These are decoupled using symmetric decoupling scheme. Due to it product of operators single out. The decoupling is done as follows:

$<<A B ; C D>>=<A C><<B ; D>>+<A D><<B ; C>>+<B C><<A ; D>>+<B D><<A ; C>>$

We obtain simple Green's functions like $<<\mathrm{A}$;B $>>,<<\mathrm{C}$; $\mathrm{D}>>$ etc. These are solved and substituted. We therefore obtain value of $\widetilde{P}(\omega)$. Resolution of $\widetilde{P}(\omega)$ into real and imaginary parts is done by following scheme:

$$
\lim _{m \rightarrow 0} \frac{1}{x+i m}=\left(\frac{1}{x}\right) \pm i \pi \delta(x)
$$


it gives shift $\Delta(\omega)$ and width $\Gamma(\omega)$ functions. We obtain:

$$
\begin{aligned}
& \Delta(\omega)=\frac{a^{4}}{\left(\omega^{2}-\tilde{\Omega}^{2}\right)}+\frac{b^{2} c^{2}}{\left(\omega^{2}-\tilde{\Omega}^{2}\right)}+\frac{V_{i k}^{2} N_{k} a^{2}}{\left(\omega^{2}-\tilde{\Omega}^{2}\right)}+\frac{2 b V_{i k}<S_{i}^{x}>\omega_{k}\left(\omega^{2}-\tilde{\tilde{\omega}}_{k}^{2}\right) \delta_{k, k^{\prime}}}{\left[\left(\omega^{2}-\tilde{\tilde{\omega}}_{k}^{2}\right)^{2}+4 \omega_{k}^{2} \Gamma_{k}^{2}(\omega)\right]}+\frac{4 b^{2} V_{i k}^{2} N_{k}}{\left(\omega^{2}-\tilde{\Omega}^{2}\right)}+\frac{8 a V_{i k}^{2}<S_{i}^{z}>\omega_{k}\left(\omega^{2}-\tilde{\tilde{\omega}}_{k}^{2}\right) \delta_{k, k^{\prime}}}{\left[\left(\omega^{2}-\tilde{\tilde{\omega}}_{k}^{2}\right)^{2}+4 \omega_{k}^{2} \Gamma_{k}^{2}(\omega)\right]} \\
& +\frac{c^{2} V_{i k}^{2} N_{k}}{\left(\omega^{2}-\widetilde{\Omega}^{2}\right)}+\frac{V_{i k}^{2} N_{k} a^{4}}{b^{2}\left(\omega^{2}-\widetilde{\Omega}^{2}\right)}+\frac{2 V_{i k}^{4} N_{k}<S_{i}^{x}>\omega_{k}\left(\omega^{2}-\tilde{\tilde{\omega}}_{k}^{2}\right) \delta_{k, k^{\prime}}}{\Omega\left(\left(\omega^{2}-\tilde{\tilde{\omega}}_{k}^{2}\right)^{2}+4 \omega_{k}^{2} \Gamma_{k}^{2}(\omega)\right]}+\frac{6 V_{i k}^{4} N_{k}<S_{i}^{z}>\omega_{k} a\left(\omega^{2}-\tilde{\tilde{\omega}}_{k}^{2}\right) \delta_{k, k^{\prime}}}{b \Omega\left[\left(\omega^{2}-\tilde{\tilde{\omega}}_{k}^{2}\right)^{2}+4 \omega_{k}^{2} \Gamma_{k}^{2}(\omega)\right.}
\end{aligned}
$$

and,

$$
\begin{aligned}
& \Gamma(\omega)=\frac{a^{4}}{2 \Omega}\{\delta(\omega-\tilde{\Omega})-\delta(\omega+\tilde{\Omega})\}+\frac{b^{2} c^{2}}{2 \widetilde{\Omega}}\{\delta(\omega-\tilde{\Omega})-\delta(\omega+\tilde{\Omega})\}+\frac{V_{i k}^{2} N_{k} a^{2}}{2 \tilde{\Omega}}\{\delta(\omega-\tilde{\Omega})-\delta(\omega+\tilde{\Omega})\} \\
& +\frac{2 b V_{i k}<S_{i}^{x}>\omega_{k} \delta_{k, k}\left\{2 \omega_{k} \Gamma_{k}(\omega)\right\}}{\left[\left(\omega^{2}-\widetilde{\widetilde{\omega}}_{k}^{2}\right)^{2}+4 \omega_{k}^{2} \Gamma_{k}^{2}(\omega)\right]}+\frac{4 b^{2} V_{i k}^{2} N_{k}}{2 \tilde{\Omega}}\{\delta(\omega-\tilde{\Omega})-\delta(\omega+\tilde{\Omega})\}+\frac{8 a V_{i k}^{2}<S_{i}^{z}>\omega_{k} \delta_{k, k} 2 \omega_{k} \Gamma_{k}(\omega)}{\left[\left(\omega^{2}-\tilde{\widetilde{\omega}}_{k}^{2}\right)^{2}+4 \omega_{k}^{2} \Gamma_{k}^{2}(\omega)\right]} \\
& +\frac{c^{2} V_{i k}^{2} N_{k}}{2 \widetilde{\Omega}}\{\delta(\omega-\tilde{\Omega})-\delta(\omega+\tilde{\Omega})\}+\frac{V_{i k}^{2} N_{k} a^{4}}{2 \widetilde{\Omega} b^{2}}\{\delta(\omega-\tilde{\Omega})-\delta(\omega+\tilde{\Omega})\} \\
& +\frac{2 V_{i k}^{4} N_{k}<S_{i}^{x}>\omega_{k} \delta_{k, k} 2 \omega_{k} \Gamma_{k}(\omega)}{\Omega\left[\left(\omega^{2}-\tilde{\widetilde{\omega}}_{k}^{2}\right)^{2}+4 \omega_{k}^{2} \Gamma_{k}^{2}(\omega)\right]}+\frac{6 V_{i k}^{4} N_{k}<S_{i}^{z}>\omega_{k} \delta_{k, k} a 2 \omega_{k} \Gamma_{k}(\omega)}{b \Omega\left[\left(\omega^{2}-\widetilde{\widetilde{\omega}}_{k}^{2}\right)^{2}+4 \omega_{k}^{2} \Gamma_{k}^{2}(\omega)\right]}
\end{aligned}
$$

In Eqs(13) and (14) $\tilde{\widetilde{\omega}}_{k}$ and $\Gamma_{k}(\omega)$ are phonon frequency and phonon width respectively. These are obtained when we solve $<<A_{k} ; A_{k},>>$ Green's function. This appear when we decouple $<<\mathrm{F}(\mathrm{t}) ; \mathrm{F}\left(\mathrm{t}^{\prime}\right)>>$ for obtaining value of $\tilde{P}(\omega)$. Phonon Green's function is obtained as:

$$
<<A_{k}(t) ; A_{k^{\prime}}^{+}\left(t^{\prime}\right)>>=\frac{\omega_{k} \delta_{k k^{\prime}}}{\pi\left[\omega^{2}-\widetilde{\widetilde{\omega}}_{k}^{2}-2 i \omega_{k} \Gamma_{k}(\omega)\right]}
$$

with,

$$
\tilde{\widetilde{\omega}}_{k}^{2}=\tilde{\omega}_{k}^{2}+2 \omega_{k} \Delta_{k}(\omega)
$$

The phonon shift $\Delta_{k}(\omega)$ is obtained as:

$$
\begin{aligned}
& \Delta_{k}(\omega)=\operatorname{Re} P_{k}(\omega)=18 P \sum_{k_{1} k_{2}}\left|V^{(3)}\left(k_{1}, k_{2},-k\right)\right|^{2} \times \frac{\omega_{k_{1}} \omega_{k_{2}}}{\tilde{\omega}_{k_{1}} \tilde{\omega}_{k_{2}}}\left\{\begin{array}{l}
\left(n_{k_{1}}+n_{k_{2}}\right) \frac{\tilde{\omega}_{k_{1}}+\tilde{\omega}_{k_{2}}}{\omega^{2}-\left(\tilde{\omega}_{k_{1}}+\tilde{\omega}_{k_{2}}\right)^{2}} \\
\left(n_{k_{2}}-n_{k_{1}}\right) \frac{\tilde{\omega}_{k_{1}}-\tilde{\omega}_{k_{2}}}{\omega^{2}-\left(\tilde{\omega}_{k_{1}}-\tilde{\omega}_{k_{2}}\right)^{2}}
\end{array}\right\} \\
& +48 P \sum_{k_{1} k_{2} k_{3}}\left|V^{(4)}\left(k_{1}, k_{2}, k_{3},-k\right)\right|^{2} \frac{\omega_{k_{1}} \omega_{k_{2}} \omega_{k_{3}}}{\widetilde{\omega}_{k_{1}} \widetilde{\omega}_{k_{2}} \tilde{\omega}_{k_{3}}} \times\left\{\begin{array}{l}
\left(1+n_{k_{1}} n_{k_{2}}+n_{k_{2}} n_{k_{3}}+n_{k_{3}} n_{k_{1}}\right) \frac{\omega_{k_{1}}+\omega_{k_{2}}+\omega_{k_{3}}}{\omega^{2}-\left(\tilde{\omega}_{k_{1}}+\tilde{\omega}_{k_{2}}+\tilde{\omega}_{k_{3}}\right)^{2}} \\
+3\left(1-n_{k_{1}} n_{k_{2}}+n_{k_{2}} n_{k_{3}}-n_{k_{3}} n_{k_{1}}\right) * \frac{\omega_{k_{1}}-\omega_{k_{2}}-\omega_{k_{3}}}{\omega^{2}-\left(\tilde{\omega}_{k_{1}}-\tilde{\omega}_{k_{2}}-\tilde{\omega}_{k_{3}}\right)^{2}}
\end{array}\right.
\end{aligned}
$$

+ higher terms .

and the phonon width $\Gamma_{k}(\omega)$ is: 


$$
\begin{aligned}
& \Gamma_{k}(\omega)=\operatorname{Im} P_{k}(\omega)=\left.9 \pi \sum_{k_{1} k_{2}} V^{(3)}\left(k_{1}, k_{2},-k\right)\right|^{2} \times \frac{\omega_{k_{1}} \omega_{k_{2}}}{\tilde{\omega}_{k_{1}} \tilde{\omega}_{k_{2}}}\left\{\begin{array}{l}
\left(n_{k_{1}}+n_{k_{2}}\right) \\
{\left[\begin{array}{l}
\delta\left(\omega+\tilde{\omega}_{k_{1}}+\tilde{\omega}_{k_{2}}\right) \\
-\delta\left(\omega-\tilde{\omega}_{k_{1}}+\tilde{\omega}_{k_{2}}\right)
\end{array}\right]} \\
\left(n_{k_{2}}-n_{k_{1}} \begin{array}{l}
\delta\left(\omega+\tilde{\omega}_{k_{1}}-\tilde{\omega}_{k_{2}}\right) \\
-\delta\left(\omega-\tilde{\omega}_{k_{1}}+\tilde{\omega}_{k_{2}}\right)
\end{array}\right]
\end{array}\right\} \\
& +48 \pi \sum_{k_{1} k_{2} k_{3}}\left|V^{(4)}\left(k_{1}, k_{2}, k_{3}, k_{4}\right)\right|^{2} \times\left[\begin{array}{l}
\delta\left(\omega+\tilde{\omega}_{k_{1}}+\tilde{\omega}_{k_{2}}+\tilde{\omega}_{k_{3}}\right) \\
-\delta\left(\omega-\tilde{\omega}_{k_{1}}-\tilde{\omega}_{k_{2}}-\tilde{\omega}_{k_{3}}\right)
\end{array}\right]+\text { higher terms. } \\
& \times\left[\left(1+n_{k_{1}} n_{k_{2}}+n_{k_{2}} n_{k_{3}}+n_{k_{3}} n_{k_{1}}\right) \frac{\omega_{k_{1}} \omega_{k_{2}} \omega_{k_{3}}}{\tilde{\omega}_{k_{1}} \tilde{\omega}_{k_{2}} \tilde{\omega}_{k_{3}}}\right]
\end{aligned}
$$

The Green's function (6) finally takes value:

$$
G_{i j}^{(\omega)}=\frac{\Omega<S_{i}^{x}>\delta_{i j}}{\pi\left[\omega^{2}-\hat{\Omega}^{2}-2 \Omega i \Gamma(\omega)\right]}
$$

Where, $\quad \hat{\Omega}^{2}=\tilde{\Omega}^{2}+\Delta(\omega)$

\section{Ferroelectric mode frequency}

Cochran [26] has suggested that ferroelectric transition occours due to becoming small of one of normal mode frequency of crystal at transition temperature. Solving $\mathrm{Eq}(20)$ we obtain:

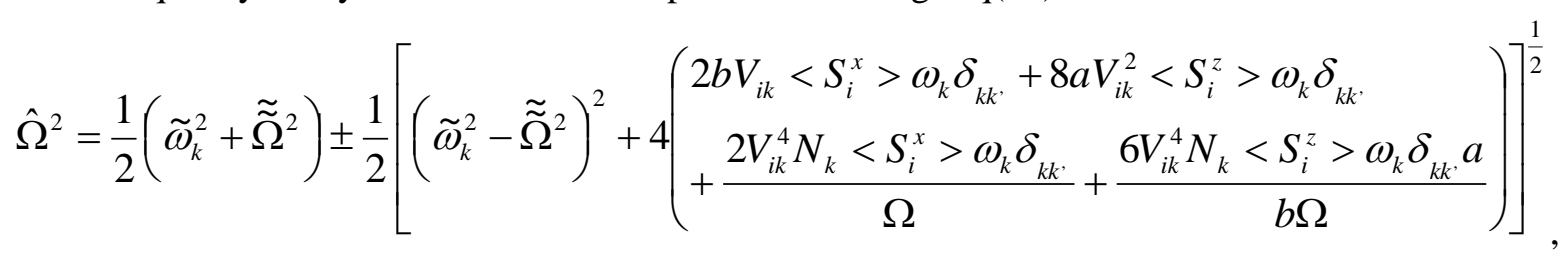

Frequency corresponding to negative sign is the ferroelectric mode frequency of MASD alum.

\section{Curie Temperature}

At transition or Curie temperature $\left(\mathrm{T}_{\mathrm{c}}\right) \widetilde{\Omega} \rightarrow 0$, so that we obtain from $\mathrm{Eq}(8)$

$$
T_{c}=\frac{\Omega}{2 k_{B} \tanh ^{-1}\left(\frac{4 \Omega}{J}\right)}
$$

where,

$$
J^{\prime}=J+\frac{2 V_{i k}^{2} \omega_{k}^{2}}{\widetilde{\widetilde{\omega}}_{k}^{2}}
$$

\section{Dielectric Constant}


Following Zubarev [25] we have:

$\chi=-\lim _{x \rightarrow 0} 2 \pi N \mu^{2} G_{i j}(\omega)$

$\chi$ is related to dielectric constant $\varepsilon$ as:

$\varepsilon=1+4 \pi \chi$.

From Eqs(19),(24) and(25) we obtain:

$\varepsilon=\frac{8 \pi N \mu^{2} \Omega<S_{i}^{x}>}{\left[\left(\omega^{2}-\hat{\Omega}^{2}\right)-2 \Omega i \Gamma(\omega)\right]}$

\section{Dielectric loss tangent}

In all ferroelectric crystals some power is lost in the form of heat which is called loss tangent $(\tan \delta)$ :

$\tan \delta=\frac{\text { imaginary } \varepsilon}{\text { real } \varepsilon}$,

from $\mathrm{Eq}(26)$ we have

$\tan \delta=\frac{2 \Omega \Gamma(\omega)}{\left(\omega^{2}-\hat{\Omega}^{2}\right)}$.

\section{Numerical Calculations \& Results}

By substituting model values for MASD alum, given in table-1, we calculate temperature dependence of $\tilde{\Omega}, \widetilde{\widetilde{\Omega}}, \hat{\Omega}, \varepsilon, \tan \delta$. Theoretical results have been compared with experimental results of Pepinsky et al [16]. Calculated values have been shown in figures 1-3.

Table 1- Model values of physical quantities for MASD alum:

\begin{tabular}{|l|l|l|l|l|l|l|l|l|}
\hline $\begin{array}{l}\mathbf{\Omega}\left(\mathbf{c m}^{-}\right. \\
\mathbf{1})\end{array}$ & $\mathbf{J}\left(\mathbf{c m}^{-\mathbf{1}}\right)$ & $\mathbf{J}^{*}\left(\mathbf{c m}^{-\mathbf{1}}\right)$ & $\begin{array}{l}\mathbf{V}_{\text {ik }} \\
\left(\mathbf{c m}^{-\mathbf{1}}\right)\end{array}$ & $\mathbf{T}_{\mathbf{c}}(\mathbf{K})$ & $\begin{array}{l}\mathbf{\Phi}_{\mathbf{k}} \\
\left(\mathbf{c m}^{-\mathbf{1}}\right)\end{array}$ & $\mathbf{C}(\mathbf{K})$ & $\boldsymbol{\mu}^{*} \mathbf{1 0}^{\mathbf{1 8}}(\mathbf{c g s})$ & $\mathbf{A}_{\mathbf{k}}{ }^{* 10} \mathbf{1 0}^{\mathbf{1 7}}(\mathbf{e r g} / \mathbf{K})$ \\
\hline 0.11 & 366.75 & 488.99 & 60.41 & 177 & 5 & 540 & 3.513 & 37.66 \\
\hline & & & & & & & & \\
\hline
\end{tabular}




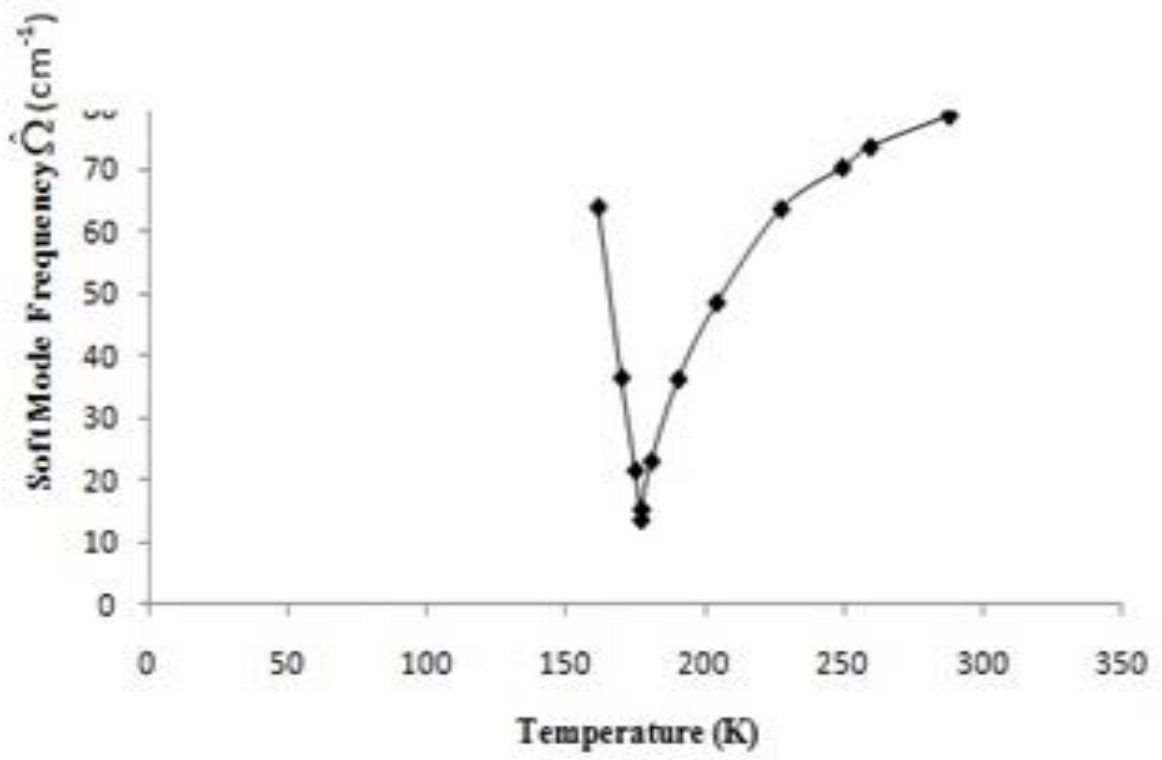

Figure 1. Temperature dependence of soft mode frequency $\hat{\Omega}\left(\mathrm{cm}^{-1}\right)$ of MASD alum (Our calculation, Correlated values with experimental values of Pepinsky et al. [16]).

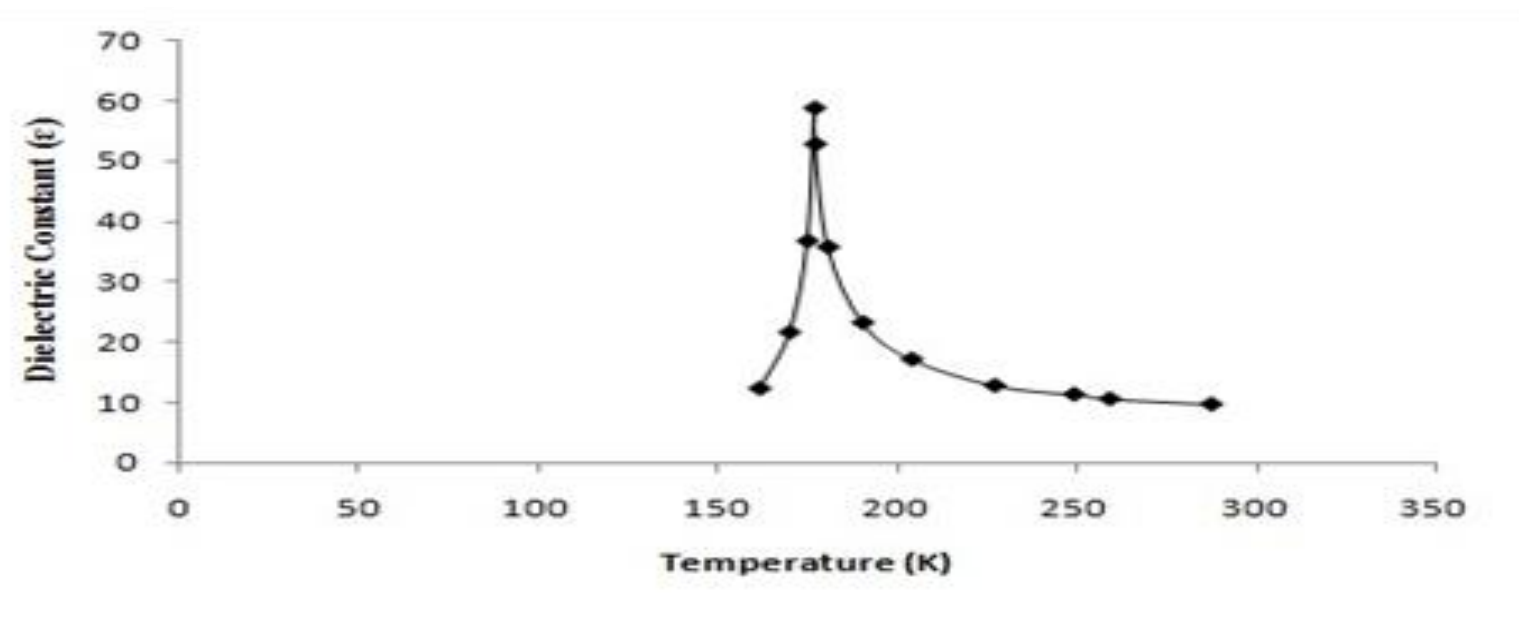

Figure 2. Temperature dependence of dielectric constant $(\varepsilon)$ of MASD alum (Our calculation, Correlated values with experimental values of Pepinsky et al. [16]). 


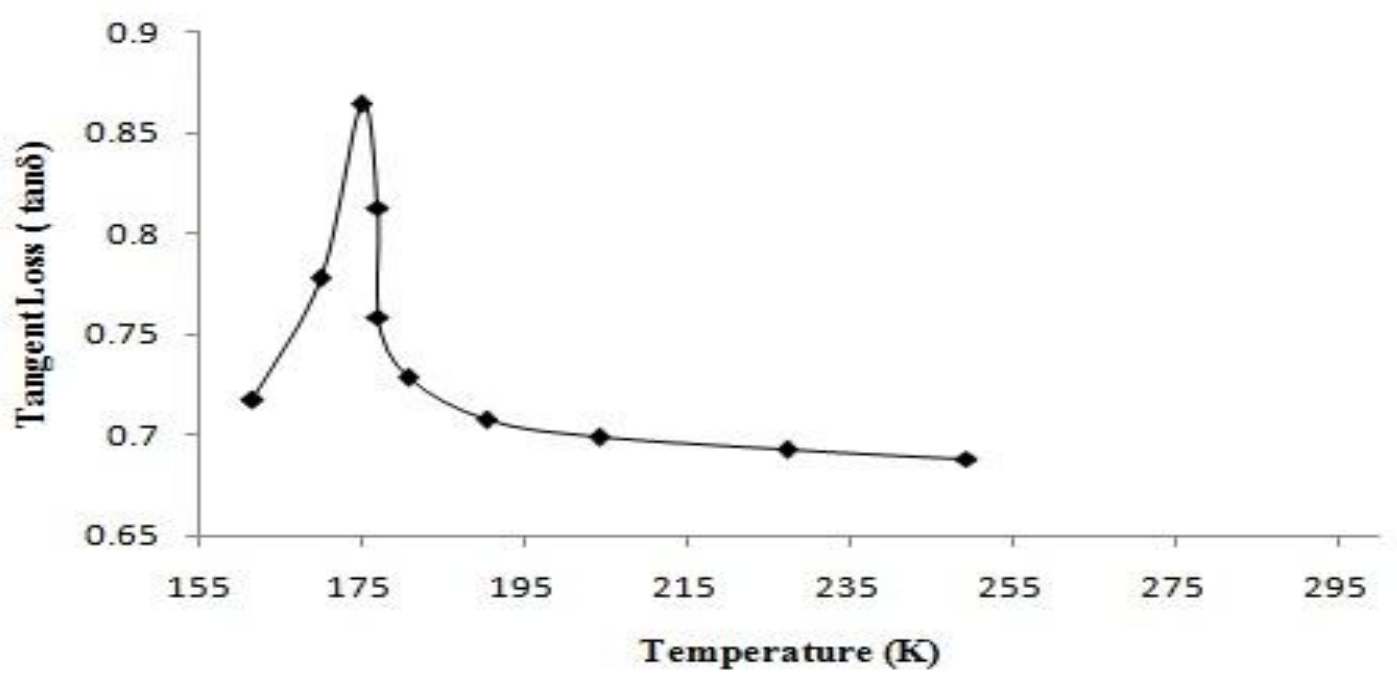

Figure 3. Temperature dependence of loss tangent $(\tan \delta)$ of MASD alum (Our calculation, Correlated with experimental values of Pepinsky et al. [16]).

\section{Discussion}

Our expression (21) for ferroelectric mode frequency shows that as temperature is increased from below $T_{c}$, the frequency decreases and becomes small at $\mathrm{T}_{\mathrm{c}}$ while above $\mathrm{T}_{\mathrm{c}}$ it increases. The phonon anharmonic terms contribute in both ferroelectric as well as paraelectric phases. Our expression (26) for dielectric constant shows that as temperature is increased from below it increases sharply becoming largest at $\mathrm{T}_{\mathrm{c}}$ and then decreases. This is due to the fact that dielectric constant is inverse squarely proportional to ferroelectric mode frequency and directly proportional to total width. Our expression (28) for loss tangent shows that as temperature is increased from below, it increases, becoming largest at $T_{c}$ and then decreases. Our predictions are in quite agreement with experimental observations for MASD alum.

\section{Conclusions}

Present study shows that modified model explains adequately the ferroelectric transition and dielectric behaviours of MASD alum. With the help of formulae obtained in present study we may explain transition and dielectric properties of similar other alums such as AFeSD, ACrSD, ANdSD alums.

\section{Acknowledgements}

Authors are greatful to Eminent Physicist Prof B.S. Semwal (Ex-Head) for his blessings and suggestions. They are thankful to Prof. S.C. Bhatt(Head), Prof. U.C. Naithani (Head, Pauri Campus), Prof. Vinay Gupta (D U), Prof. N S Negi (H P U Shimla) and Prof Shyam Kumar (Kurukshatra) for their encouragements.

\section{References}

[1] C G N Ronald, K Asadi, W M B Paul, M De L Dago and B de Bert, Adv. Mater 22933 ( 2011)

[2] G M Venkatesh and P S Narayanan, J. of the Indian Inst. of Sci. 522133 (1970)

[3] J H E Griffiths and J A Powell, Proc. Roy. Soc. London Sec. A 65289 (1952)

[4] A H Rama Rao, M R Srinivasan, H L Bhat and P S Narayanan, Ferroelectrics 21433 
[5] J J Derby, 14 ${ }^{\text {th }}$ Int'l. Summar School on Crys. Growth (ISSIG-141, Dallian Chaina 1-7 $\operatorname{Aug}(2010)$

[6] H J Weber, Zeits fur. Kvistall 149269 (2010)

[7] M Boujelben and $\mathrm{T}$ Mihiri, Int l. J. of Spectroscopy 201112840 (2011)

[8] Sachdeva et al., Materials \& Technology 4 467 (2013)

[9] H Gu and Y Li, Spectrochimia Acta Part A 139342 (2015)

[10] H Gu and X Hao, Spectrochimia Acta Part A 146273 (2015)

[11] W Zhang, C L Zhuang, R G Xiong, T Nakamura and S D Huang, J. Anul. Chem. Soc. 1311255 (2009)

[12] S M A Basara, Z Iqbal, K Rahman, H Rahman and M F Ejaz, J. Appl. Res. Tech. 12560 (2014)

[13] P K Singh, B Bhattacharya, A Sachdeva and R Singh, Mater. Tech. 47467 (2013)

[14] S G Sajan, A S Ayesh, A Zihilif, E Martuscelli and R Zagosta , Poly. Test 23739 (2004)

[15] H M E Ghanem, S Saquam, M Saadi and S Abdul Zawad, J. Mod. Phys. 21533 (2011)

[16] R Pepinsky, F Jona and G Shirane, Phys. Rev. 1021181 (1956)

[17] Yu Shaxin et al., Organic Synthesis 17 2730 (2005)

[18] V M Petrusevski, Macedonian J. of Chemistry \& Chemical Engineering 3473 (2015)

[19] M Bow and L M Ennio Gregorio, Ulmer Jeffrey B Nature Medicine 17415 (2011)

[20] A Mansingh, S B Krupanidhi and E

Prasad, J. Chem. Phys. 69(7) 3039 (1978)

[21] Y H Cha and $\mathrm{H}$ L Strauss, Inorganic Chemistry 40(6) 1176 (2001)

[22] Lou, Ssu-Hao, Jiang-Tsu, Solid State Communications, 84(7) 775 (1992)

[23] D E O' Reilly and T Tsang, Phys. Rev. 157 417 (1967)

[24] K R Chaudhury, D Nath, S Banerjee and B K Chaudhuri, Phys. Rev. B 266276 (1982)
[25] D N Zubarev, Sov. Phys. Usp. 3320 (1960)

[26] W Cochran, Phil. Mag. Suppl. 10401 (1961) 